\title{
Guidelines for Classification and Interpretation of Group Blood and Urine Data Collected as Part of the National Nutrition Survey
}

\author{
R.M.O'Neal, O.C.Johnson and A.E. SGhaefer \\ United States Department of Health, Education and Welfare, Health Services and Mental Health \\ Administration, Regional Medical Programs Service, Nutrition Program, Bethesda, Maryland, USA
}

During the past few months interest in the nutritional health status of United States populations has increased by several magnitudes. This interest has resulted in a number of studies designed to objectively ascertain the current status of nutritional health in this country. One portion of such objective evaluations usually consists of a number of biochemical determinations on blood and urine that are related to intake, excretion or storage, or both, of specific nutrients. To relate data obtained from such biochemical measurements to nutritional health status, they must be compared with biochemical values deemed to reflect various degrees

Table II. Methodology used to obtain National Nutrition Survey laboratory data

\begin{tabular}{|c|c|}
\hline Determination & Method \\
\hline Hemoglobin & Cyanmethemoglobin $^{1}$ \\
\hline Hematocrit & Micromethod ${ }^{2}$ \\
\hline Serum iron & Giovanniello ${ }^{3}$ \\
\hline Serum iron-binding & \\
\hline capacity & Caraway 4 \\
\hline Blood folacin & Lactobacillus casei $i^{5}$ \\
\hline Total serum protein & Biuret $^{6}$ \\
\hline Serum albumin & $\begin{array}{l}\text { 2-(4'-hydroxyazobenzene) } \\
\text { benzoic acid (HABA) }\end{array}$ \\
\hline Serum vitamin $G$ & $\begin{array}{l}\text { Macro dinitrophenyl- } \\
\text { hydrazine }^{1}\end{array}$ \\
\hline $\begin{array}{l}\text { Plasma carotene and } \\
\text { vitamin } \mathrm{A}\end{array}$ & $\begin{array}{l}\text { Macro Carr-Price } \\
\text { (trifluoroacetic acid } \\
\text { chromogen) }{ }^{1}\end{array}$ \\
\hline Urinary creatinine & Picrate $^{6}$ \\
\hline Urinary thiamine & Thiochrome $^{1}$ \\
\hline Urinary riboflavin & Modified Slater-Morell ${ }^{1}$ \\
\hline Urinary iodine & Modified Zak ${ }^{1}$ \\
\hline
\end{tabular}

of nutritional health status. Such a set of values has been developed to interpret the data currently being collected as part of the National Nutrition Survey (NNS). Since it is a national survey and the results obtained will be used by numerous groups concerned with nutritional health and for comparison with other nutritional health studies, the guidelines developed for classification and interpretation of group blood and urine data collected as part of the NNS should be made available to interested groups.

Prior to the beginning of the NNS, considerable amounts of information were available relating biochemical measurements to dietary and clinical nutrition status, but a current set of comprehensive guidelines suitable for interpretation of the biochemical data generated by the NNS was not available. The guidelines that had been suggested for interpretation

\footnotetext{
${ }^{1}$ Manual for nutrition surveys, 2nd ed., Interdepartmental Committee on Nutrition for National Defense, 1963.

${ }^{2}$ M.M.Wintrobe: Clinical hematology, 6th ed. (Lea \& Febiger, Philadelphia 1967).

${ }^{3}$ Modified procedure: Giovanniello, T.J.; DiBenedetto, G.; Palmer, D.W. and Peters, T., Jr.: Fully and semi-automated methods for the determination of serum iron and total iron-binding capacity. J. Lab. Clin. Med. 71: 874 (1968).

${ }^{4}$ Modified procedure: Caraway, W.T.: Macro and micro methods for the determination of serum iron and iron-binding capacity. Clin. Chem.9: 188 (1963).

${ }^{5}$ Modified by U.S. Army Medical Research and Nutrition Laboratory, Fitzsimons General Hospital, Denver, Colo.

${ }^{6}$ Technicon auto analyzer methodology, Technicon Instruments Corporation, Chauncey, N.Y.
} 
Table I. Guidelines for classification and interpretation of group blood and urine data collected as part of the National Nutrition Survey

\begin{tabular}{|c|c|c|c|}
\hline \multirow[t]{3}{*}{ Determination } & \multicolumn{3}{|c|}{ Classification category } \\
\hline & \multicolumn{2}{|c|}{ Less than acceptable } & \multirow[t]{2}{*}{ Acceptable ${ }^{1}$} \\
\hline & Deficient & Low & \\
\hline \multicolumn{4}{|l|}{ Hemoglobin, $\mathrm{g} / 100 \mathrm{ml}$} \\
\hline $6-23$ months & $<9.0$ & $9.0-9.9$ & $\geqq 10.0$ \\
\hline $2-5 \mathrm{yr}$ & $<10$ & $10.0-10.9$ & $\geqq 11.0$ \\
\hline $6-12 \mathrm{yr}$ & $<10$ & $10.0-11.4$ & $\geqq 11.5$ \\
\hline $13-16$ yr, male & $<12$ & $12.0-12.9$ & $\geqq 13.0$ \\
\hline $13-16 \mathrm{yr}$, female & $<10$ & $10.0-11.4$ & $\geqq 11.5$ \\
\hline$>16 \mathrm{yr}$, male & $<12$ & $12.0-13.9$ & $\geqq 14.0$ \\
\hline$>16 \mathrm{yr}$, female & $<10$ & $10.0-11.9$ & $\geqq 12.0$ \\
\hline Pregnant, 2nd trimester & $<9.5$ & $9.5-10.9$ & $\geqq 11.0$ \\
\hline Pregnant, 3rd trimester & $<9.0$ & $9.0-10.4$ & $\geqq 10.5$ \\
\hline \multicolumn{4}{|l|}{ Hematocrit, $\%$} \\
\hline 6-23 months & $<28$ & $28-30$ & $\geqq 31$ \\
\hline $2-5 \mathrm{yr}$ & $<30$ & $30-33$ & $\geqq 34$ \\
\hline $6-12$ yr & $<30$ & $30-35$ & $\geqq 36$ \\
\hline $13-16 \mathrm{yr}$, male & $<37$ & $37-39$ & $\geqq 40$ \\
\hline $13-16$ yr, female & $<31$ & $31-35$ & $\geqq 36$ \\
\hline$>16 \mathrm{yr}$, male & $<37$ & $37-43$ & $\geqq 44$ \\
\hline$>16 \mathrm{yr}$, female & $<31$ & $31-37$ & $\geqq 38$ \\
\hline Pregnant, 2nd trimester & $<30$ & $30-34$ & $\geqq 35$ \\
\hline Pregnant, 3rd trimester & $<30$ & $30-32$ & $\geqq 33$ \\
\hline \multicolumn{4}{|c|}{ Hemoglobin conc, MCHC, $\mathrm{g} / 100 \mathrm{ml} \mathrm{RBC}$} \\
\hline All ages & - & 30 & $\geqq 30$ \\
\hline \multicolumn{4}{|l|}{ Serum iron, $\mu \mathrm{g} / 100 \mathrm{ml}$} \\
\hline $0-5$ months & & & - \\
\hline $6-23$ months & & & $\geqq 30$ \\
\hline $2-5 \mathrm{yr}$ & & & $\geqq 40$ \\
\hline $6-12 \mathrm{yr}$ & & & $\geqq 50$ \\
\hline$>12$ yr, male & & & $\geqq 60$ \\
\hline$>12 \mathrm{yr}$, female & & & $\geqq 40$ \\
\hline \multicolumn{4}{|l|}{ Transferrin saturation, $\%$} \\
\hline $0-5$ months & & & - \\
\hline $6-23$ months & & & $\geqq 15$ \\
\hline $2-12 \mathrm{yr}$ & & & $\geqq 20$ \\
\hline$>12 \mathrm{yr}$, male & & & $\geqq 20$ \\
\hline$>12$ yr, female & & & $\geqq 15$ \\
\hline \multicolumn{4}{|l|}{ Red cell folacin, $\mathrm{ng} / \mathrm{ml}$} \\
\hline All ages & $<140$ & $140-159$ & $\geqq 160-650$ \\
\hline Serum folacin, $\mathrm{ng} / \mathrm{ml}$ & 3.0 & $3.0-5.9$ & $\geqq 6.0$ \\
\hline \multicolumn{4}{|l|}{ Serum protein, g/100 ml } \\
\hline 0-11 months & & $<5.0$ & $\geqq 5.0$ \\
\hline $1-5 \mathrm{yr}$ & & $<5.5$ & $\geqq 5.5$ \\
\hline $6-17 \mathrm{yr}$ & & $<6.0$ & $\geqq 6.0$ \\
\hline Adult & $<6.0$ & $6.0-6.4$ & $\geqq 6.5$ \\
\hline Pregnant, 2nd and 3rd trimester & $<5.5$ & $5.5-5.9$ & $\geqq 6.0$ \\
\hline
\end{tabular}


Table I. (Continued)

\begin{tabular}{|c|c|c|c|}
\hline \multirow[t]{3}{*}{ Determination } & \multicolumn{3}{|c|}{ Classification category } \\
\hline & \multicolumn{2}{|c|}{ Less than acceptable } & \multirow[t]{2}{*}{ Acceptable ${ }^{1}$} \\
\hline & Deficient & Low & \\
\hline \multicolumn{4}{|l|}{ Serum albumin, $\mathrm{g} / 100 \mathrm{ml}$} \\
\hline $0-11$ months & & $<2.5$ & $\geqq 2.5$ \\
\hline $1-5 \mathrm{yr}$ & & $<3.0$ & $\geqq 3.0$ \\
\hline $6-17$ yr & & $<3.5$ & $\geqq 3.5$ \\
\hline Adult & $<2.8$ & $2.8-3.4$ & $\geqq 3.5$ \\
\hline Pregnant, 1st trimester & $<3.0$ & $3.0-3.9$ & $\geqq 4.0$ \\
\hline Pregnant, 2 nd and 3rd trimester & $<3.0$ & $3.0-3.4$ & $\geqq 3.5$ \\
\hline \multicolumn{4}{|l|}{ Serum vitamin $\mathrm{C}, \mathrm{mg} / 100 \mathrm{ml}$} \\
\hline $0-11$ months & - & - & - \\
\hline$\geqq 1 \mathrm{yr}$ & $<0.1$ & $0.1-0.19$ & $\geqq 0.2$ \\
\hline \multicolumn{4}{|l|}{ Plasma carotene, $\mu \mathrm{g} / 100$} \\
\hline $0-5$ months & & $<10$ & $\geqq 10$ \\
\hline $6-11$ months & & $<30$ & $\geqq 30$ \\
\hline $1-17 \mathrm{yr}$ & & $<40$ & $\geqq 40$ \\
\hline Adult & $<20^{2}$ & $20-39$ & $\geqq 40$ \\
\hline Pregnant, 2nd trimester & & $30-79$ & $\geqq 80$ \\
\hline Pregnant, 3rd trimester & & $40-79$ & $\geqq 80$ \\
\hline \multicolumn{4}{|l|}{ Plasma vitamin $\mathrm{A}, \mu \mathrm{g} / 100 \mathrm{ml}$} \\
\hline $0-5$ months & $<10$ & $10-19$ & $\geqq 20$ \\
\hline $0.5-17 \mathrm{yr}$ & $<20$ & $20-29$ & $\geqq 30$ \\
\hline Adult & $<10$ & $10-19$ & $\geqq 20$ \\
\hline \multicolumn{4}{|l|}{ Urinary thiamine, $\mu \mathrm{g} / \mathrm{g}$ creatinine } \\
\hline $1-3 \mathrm{yr}$ & $<120$ & $120-175$ & $\geqq 176$ \\
\hline $4-6 y r$ & $<85$ & $85-120$ & $\geqq 121$ \\
\hline $7-9 \mathrm{yr}$ & $<70$ & $70-180$ & $\geqq 181$ \\
\hline $10-12 \mathrm{yr}$ & $<60$ & $60-180$ & $\geqq 181$ \\
\hline $13-15 \mathrm{yr}$ & $<50$ & $50-150$ & $\geqq 151$ \\
\hline Adult & $<27$ & $27-65$ & $\geqq 66$ \\
\hline Pregnant, 2nd trimester & $<23$ & $23-54$ & $\geqq 55$ \\
\hline Pregnant, 3rd trimester & $<21$ & $21-49$ & $\geqq 50$ \\
\hline \multicolumn{4}{|l|}{ Urinary riboflavin, $\mu \mathrm{g} / \mathrm{g}$ creatinine } \\
\hline $1-3 \mathrm{yr}$ & $<150$ & $150-499$ & $\geqq 500$ \\
\hline $4-6 y r$ & $<100$ & $100-299$ & $\geqq 300$ \\
\hline $7-9 \mathrm{yr}$ & $<85$ & $85-269$ & $\geqq 270$ \\
\hline $10-15 \mathrm{yr}$ & $<70$ & $70-199$ & $\geqq 200$ \\
\hline Adult & $<27$ & $27-79$ & $\geqq 80$ \\
\hline Pregnant, 2nd trimester & $<39$ & $39-119$ & $\geqq 120$ \\
\hline Pregnant, 3rd trimester & $<30$ & $30-89$ & $\geqq 90$ \\
\hline Urinary iodine, $\mu \mathrm{g} / \mathrm{g}$ creatinine & $<25$ & $25-49$ & $\geqq 50$ \\
\hline
\end{tabular}

${ }^{1}$ Excessively high levels may indicate abnormal clinical status or toxicity.

${ }^{2}$ May indicate unusual diet or malabsorption. 
of biochemical data by the Interdepartmental Committee on Nutrition for National Defense (ICNND) [2] were selected as a starting point and revised and expanded to accommodate the scope of data collected from the NNS. The Committee on Nutrition of the American Academy of Pediatrics reviewed the ICNND guidelines for interpretation of blood data, and revised them when necessary, based on current information [1]. The Committee expanded them to accommodate the significant physiological differences in children due to sex and age through 16 years of age, as well as providing guidelines for additional determinations performed in the NNS.

The Nutrition Program Advisory Committee reviewed the ICNND guidelines for interpretation of blood and urine data for adults and revised them where current information required revision. This committee expanded these guidelines to include standards for interpretation of additional determinations performed in the NNS.

The comprehensive guidelines for classification and interpretation of group blood and urine data collected as part of the NNS are shown in table I and are for laboratory data obtained by the methods referenced in table II.

\section{References and Notes}

1. Diseases related to nutrition, rates and rank order; Systems Development Project Staff, Minneapolis, Minnesota, 1968.

2. Manual for nutrition surveys, 2nd ed., Interdepartmental Committee on Nutrition for National Defense, 1963.

3. Accepted for publication October 13, 1969. 